Trinity University

Digital Commons @ Trinity

Physics and Astronomy Faculty Research

Physics and Astronomy Department

2006

\title{
Energetics of Magnetic Storms Driven by Corotating Interaction Regions: A Study of Geoeffectiveness
}

\author{
Niescja E. Turner \\ Trinity University, nturner1@trinity.edu \\ E J. Mitchell \\ D J. Knipp
}

B A. Emery

Follow this and additional works at: https://digitalcommons.trinity.edu/physics_faculty

Part of the Astrophysics and Astronomy Commons

\section{Repository Citation}

Turner, N. E., Mitchell, E. J., Knipp, D. J., \& Emery, B. A. (2006). Energetics of magnetic storms driven by corotating interaction regions: A study of geoeffectiveness. In B. Tsurutani, R. McPherron, G. Lu, J. H. A. Sobral, \& N. Gopalswamy (Eds.), Recurrent magnetic storms: Corotating solar wind streams (vol. 167, pp. 113-124). American Geophysical Union.

This Contribution to Book is brought to you for free and open access by the Physics and Astronomy Department at Digital Commons @ Trinity. It has been accepted for inclusion in Physics and Astronomy Faculty Research by an authorized administrator of Digital Commons @ Trinity. For more information, please contact jcostanz@trinity.edu. 


\title{
Energetics of Magnetic Storms Driven by Corotating Interaction Regions: A Study of Geoeffectiveness
}

\author{
Niescja E. Turner and Elizabeth J. Mitchell \\ Florida Institute of Technology, Melbourne, Florida \\ Delores J. Knipp \\ United States Air Force Academy, Colorado Springs, Colorado
}

\author{
Barbara A. Emery
}

High Altitude Observatory, National Center for Atmospheric Research, Boulder, Colorado

We investigate the energetics of magnetic storms associated with corotating interaction regions (CIRs). We analyze 24 storms driven by CIRs and compare to 18 driven by ejecta-related events to determine how they differ in overall properties and in particular in their distribution of energy. To compare these different types of events, we look at events with comparable input parameters such as the epsilon parameter and note the properties of the resulting storms. We estimate the energy output by looking at the ring current energy along with ionospheric Joule heating derived from the PC and Dst indices. We also include the energy of auroral precipitation, estimated from NOAA/TIROS and DMSP observations. In general, ejecta-driven storms produce more intense events, as parameterized by $D s t^{*}$, but they are usually not as long lasting, and in most cases deposit less energy. This is observed even for events that have similar input quantities, such as epsilon. This may be related to the high speed of the solar wind, in that an increased magnetosonic Mach number may influence the reconnection rate and therefore the coupling. Additionally, we find the efficiency of the coupling varies greatly from CIR-driven to ejecta-driven storms, with the CIRdriven storms coupling substantially more efficiently, particularly in the recovery phase. The efficiency of coupling (output energy divided by input energy) for CIRdriven storms in recovery phase was double that of ejecta-driven storms.

\section{INTRODUCTION}

\subsection{Geoeffectiveness}

Geoeffectiveness refers to the efficiency of energy coupling from the solar wind into the magnetosphere. It can be

Recurrent Magnetic Storms: Corotating Solar Wind Streams Geophysical Monograph Series 167

Copyright 2006 by the American Geophysical Union.

10.1029/167GM11 estimated by looking at the solar wind input and the corresponding magnetospheric output for a particular time period. The question becomes, then, how does one quantify the solar wind input and the magnetospheric output? Solar wind input has been parameterized in several key ways over the years, usually in the form of a Poynting flux, and this remains the most widespread estimate. Magnetospheric output, however, is less clear-cut. Some use widely known magnetospheric activity indices such as $D s t$ or $K p$ to estimate the response to solar wind drivers. Other researchers may be more interested in the radiation belt response, for example, and have different 
approaches to quantifying that response. Since the radiation belts are less significant energetically than other sinks, they are not included in this study. For the purposes of this paper, magnetospheric response will be regarded as the total amount of energy (or, in some cases, the instantaneous power) being deposited into the primary magnetospheric energy sinks. These sinks include the ring current, ionospheric Joule heating, and auroral precipitation. As will become clear, this definition of magnetospheric response will often produce different results than other definitions, especially when it comes to defining the types of solar wind drivers that are most geoeffective.

\subsection{Magnetospheric Energy Input}

Precise measurements of the total amount of energy entering the magnetosphere from the solar wind are simply not possible. Over the years, however, estimates have been made. The epsilon parameter is one such estimate. Epsilon is defined (in SI units) as:

$$
\varepsilon=\left(4 \pi / \mu_{0}\right) v B^{2} \sin ^{4}(\theta / 2) l_{0}^{2}
$$

where $v$ is the solar wind speed, $B$ is the magnitude of the IMF, $l_{0}$ is a characteristic length scale representing the coupling area available for solar wind-magnetosphere interactions, usually approximated as $7 \mathrm{R}_{\mathrm{E}}$, [Perreault and Akasofu, 1978], $\mu_{0}$ is the permeability of free space, and $\theta$ is defined as $\tan ^{-1}\left(\left|B_{Y}\right| / B_{Z}\right)$. Epsilon is a measure of the Poynting flux in the solar wind over the magnetospheric collecting area. It uses a "leaky" filter of $\sin ^{4}(\theta / 2)$, which means that, while energy coupling is greatly enhanced for southward $B_{Z}$, there is still some coupling for northward $B_{Z}$ as well. The form of epsilon is based on empirical studies of the estimated energy dissipation in the magnetosphere. Thus, while the form of epsilon is shown to replicate the pattern of energy dissipation, its scaling should be considered somewhat arbitrary, as it is based on estimates of energy output rather than quantitative knowledge of energy input. Additionally, the $l_{0}{ }^{2}$ term in this equation does not vary, although the magnetopause area is known to vary with solar wind conditions [see, e.g., Monreal-MacMahon and Gonzalez, 1997].

While epsilon is the most commonly used parameterization of solar wind energy input, there are others. One effort to revisit the issue of energy input into the magnetosphere was conducted by Bargatze et al. [1985]. They derived a more complex coupling equation which ultimately also used the same $\sin ^{4}(\theta / 2)$ term and was similar in form to the epsilon parameter.

Other researchers parameterize solar wind energy input with $v B_{S}$, where $\mathrm{v}$ is the solar wind velocity and $B_{S}$ is the southward component of the IMF [e.g., O'Brien and
McPherron, 2000], or would include the solar wind kinetic energy flux [e.g., Lu et al., 1998]. It is likely that the actual coupling involves some combination of these, and certain parameters may become more or less dominant under different conditions. Since the kinetic energy flux is large in comparison with the epsilon-derived energy input, it would not require a very strong coupling in this regard to have a pronounced effect on the magnetosphere.

\section{PREVIOUS WORK}

Many researchers have investigated the flow of energy in the magnetosphere [e.g., Turner et al., 2000b; Baker et al., 2001; Weiss et al., 1992; Vichare et al., 2005]. Lu et al. [1998] investigated energy budgets in the magnetic storm interval that occurred in January of 1997. They used Assimilative Mapping of Ionospheric Electrodynamics (AMIE) calculations to determine the energy lost to ionospheric processes, and used the standard Dst index to estimate the ring current injection rate. Overall, in the January 10 and 11, 1997 case, Lu et al., estimated that the magnetosphere-ionosphere system dissipated an average of about $4.0 \times 10^{11} \mathrm{~W}$. Of this, $1.9 \times 10^{11}$ $\mathrm{W}$ (or $48 \%$ ) went into Joule heating, $1.2 \times 10^{11} \mathrm{~W}$ (or $30 \%$ ) went into ring current injection, and $0.9 \times 10^{11} \mathrm{~W}$ (or 22\%) went into auroral precipitation. They did not estimate the energy lost to plasmoids streaming down the magnetotail. Knipp et al. [1998] analyzed the November 1993 storm, which was a hybrid event where a high-speed stream followed a CME. They found that high-speed streams could be enormously geoeffective, and for this extreme event the ionospheric heating was $\sim 190 \times 10^{15} \mathrm{~J}$, with $30 \%$ of that generated within 24 hours of storm onset.

Gonzalez et al. [1989] specifically analyzed energy coupling during intense $(D s t<-100 \mathrm{nT})$ storm events and tested the responses of several coupling functions to investigate the $D s t$ response. They found that solar wind ram pressure played a role in ring current energization and that during the strong events they studied, there seemed to be a decoupling of auroral response from inner magnetospheric response for the solar wind-magnetosphere coupling functions they analyzed.

Six storms were analyzed by Turner et al. [2000b] to determine their energy input and deposition rates. Their calculation incorporated AMIE data to determine the ionospheric loss and a pressure- and tail-corrected form of Dst to track the ring current energy, and they also included a term for plasmoid ejection loss. In all cases, epsilon was shown to correlate with the energy output, and in 5 of the 6 events epsilon was estimated to be larger than the output energy. The results of this analysis showed a clear dominance of ionospheric energy deposition over other processes. In fact, Joule heating alone typically accounted for around half or more of the total energy output. The ring current contribution was less than in 
previous estimates, largely due to a reevaluation of the ring current strength compared to Dst* [Turner et al., 2001, 2000a], and also due to the AMIE analysis suggesting a larger ionospheric loss. This analysis shows the ring current energy to be approximately $10 \%-15 \%$ of the total energy output.

The polar cap (PC) index can be used as a proxy indicator describing the amount of energy deposited into the ionosphere in the form of Joule heating and auroral precipitation. Chun et al. [1999], based on comparisons with AMIE data assimilation results, have shown a quadratic relationship between the PC index and the hemispheric integrated Joule heating rate, and recent work shows a linear relationship between PC and electron precipitation. More recent work by Knipp et al. [2004] has shown a better fit to the data if both $\mathrm{PC}$ and $D s t$ are used as inputs.

The study by Turner et al. [2000b] of the global energy budget of the magnetosphere analyzed several storms over a fairly small time frame (about 2 years), so it was limited to a small portion of the solar cycle. It is known that solar wind driving conditions vary over the solar cycle, with corotating interaction regions (CIRs) being more common during solar minimum, and coronal mass ejections (CMEs) being more common toward solar maximum. Many researchers have observed differences in the dynamics of storms during times of different types of solar wind driving conditions, such as the existence of High-Intensity Long-Duration Continuous Auroral Activity (HILDCAA) events in the recovery phase of CIR-driven events [e.g., Tsurutani et al., 2004, 2006 (this volume)]. On average, CIRs have less steady $B_{Z}$ and higher bulk speed than CMEs, and the resulting storms differ in some fundamental properties. Some researchers have studied the ability of different types of solar wind structures to produce storms [see, e.g., Zhang et al., 2004]. Echer and Gonzalez [2004] found that compound interplanetary structures were more geoeffective than isolated structures. In another study, Huttunen et al. [2002] looked at storms from 1996 to 1999. They found that almost all the intense $(D s t<-100 \mathrm{nT})$ storms were associated with CMEs, but for the moderate storms, streams more often generated high $K p$ storms, while ejecta-related events more often drove stronger Dst changes. This could suggest that the relative impacts on the ring current and the ionosphere could vary by type of solar wind driver. Gonzalez et al. [1999] found that complex interplanetary structures, including in rare circumstances the influence of subsequent CMEs, could drive particularly intense geomagnetic storms.

\section{METHODOLOGY}

A list of events sorted by solar wind drivers (Ian Richardson, private communication) was analyzed. These data were classified by time intervals of CMEs and CIRs during
1995-1998. Ionospheric Joule heating power was calculated according to the relations derived by Knipp et al. [2004]. The relations for the Joule heating in GW for the northern hemisphere are:

$$
\begin{gathered}
\mathrm{JH}_{\text {summer }}=29.27|\mathrm{PC}|+8.18 \mathrm{PC}^{2}-0.04|D s t|+0.0126 D s t^{2} \\
\mathrm{JH}_{\text {equinox }}=29.14|\mathrm{PC}|+2.54 \mathrm{PC}^{2}+0.21|D s t|+0.0023 D s t^{2} \\
\mathrm{JH}_{\text {winter }}=13.36|\mathrm{PC}|+5.08 \mathrm{PC}^{2}+0.47|D s t|+0.0011 D s t^{2},
\end{gathered}
$$

where summer is defined as 21 April - 20 August, winter is 21 October -20 February, and equinox is 21 February -20 April and 21 August -20 October. For equinox times, northern hemisphere values were doubled to obtain a global value. For summer and winter dates, a Joule heating estimate for summer was added to a winter estimate to account for the hemispheric seasonal differences.

Global auroral precipitation values were computed from NOAA/TIROS and DMSP satellite measurements of highlatitude precipitating energy flux carried by ions and electrons with energies between $300 \mathrm{eV}$ and $20 \mathrm{keV}$ (NOAA/TIROS) or carried by electrons with energies between $460 \mathrm{eV}$ and $30 \mathrm{keV}$ (DMSP). The energy flux observations made during a single pass over the polar regions are used to estimate the total precipitating power input to a single hemisphere at that time. The power index was devised by Dave Evans for NOAA/TIROS and adapted for DMSP by Frederick Rich and William Denig [Emery et al., 2005, 2006]. Global values were calculated by adding a southern hemisphere estimate to a northern hemisphere estimate.

All solar wind data used in this study were offset to allow propagation time from the satellite to the magnetopause.

The Dst index was pressure corrected to $D s t^{*}$ using the Burton et al. [1975] equation, $D s t^{*}=D s t-\mathrm{b} P^{1 / 2}+\mathrm{c}$, where $P$ is the solar wind dynamic pressure and the constants $\mathrm{b}$ and $\mathrm{c}$ are $\mathrm{b}=7.26$ and $\mathrm{c}=11.0$ as derived by $O^{\prime}$ Brien and McPherron [2000].

After pressure correction, the ring current energy was estimated using $D s t^{*}$. As many researchers have pointed out, [e.g., Campbell, 1996], the Dst index measures the effects of many key current systems and cannot single out the ring current. Magnetotail currents are among the primary current systems that can perturb the Dst index [see Turner et al., 2000a; Ohtani et al., 2001; Feldstein et al., 2005], as well as induced ground currents. $D s t^{*}$ was corrected to account for these using the relation described in Turner et al. [2001]. This calculation is made by halving the (pressure-corrected) $D s t *$ to remove the influence of induced ground currents and tail currents and then applying the standard Dessler-ParkerSckopke [Dessler and Parker, 1959; Sckopke, 1966] relation. The Dessler-Parker-Sckopke (DPS) relationship between global magnetic field perturbation and particle energy is as 
follows: $\Delta \mathrm{B}=-\left(\mu_{0} / 2 \pi\right) \mathrm{W}_{\text {particles }} /\left(\mathrm{B}_{0} \mathrm{R}_{\mathrm{E}}^{3}\right)$, where $\Delta \mathrm{B}$ is approximated by the corrected $D s t, \mathrm{~W}_{\text {particles }}$ is the particle energy, $\mu_{0}$ is the permeability of free space, $\mathrm{B}_{0}$ is the surface dipole strength at the equator, and $\mathrm{R}_{\mathrm{E}}$ is the radius of the Earth.

So, to calculate the ring current energy from Dst, the Dst index was first pressure corrected, then halved, and then plugged into the DPS relation to solve for the particle energy. The ring current injection rate was then calculated using the relationship derived by Akasofu [1981], Q $=-4 \times 10^{13}$ $(\partial D s t / \partial \mathrm{t}+D s t / \tau)$ in SI units, with the ring current decay time set to $\tau=6$ hours. The epsilon parameter was used to estimate energy input.

Storm intervals were identified where $D s t^{*}$ was less than $-50 \mathrm{nT}$ and a storm recovery phase was observed with at least $80 \% D s t^{*}$ recovery. From these criteria, 24 CIR-driven storms and 18 ejecta-driven storms were identified and analyzed. For a complete list of events in this study, see Tables 1 and 2 .

Two example events are shown in Figure 1. A CIR-driven event from April 16-21, 1997 is shown to scale alongside a CME-driven event from April 21-23, 1997. These events are representative of the population of storms in this study with the CIR-driven event lasting approximately 2.75 times longer

Table 1. List of all CIR-driven events in the study

\begin{tabular}{|c|c|c|c|c|}
\hline $\begin{array}{l}\text { Start } \\
\text { Date }\end{array}$ & Hour & $\begin{array}{l}\text { End } \\
\text { Date }\end{array}$ & Hour & $\begin{array}{c}\text { Minimum } \\
\text { Dst* }\end{array}$ \\
\hline 29-Jan-95 & 9 & 5-Feb-95 & 5 & -74.22 \\
\hline $11-F e b-95$ & 6 & $16-F e b-95$ & 22 & -52.13 \\
\hline 7-Apr-95 & 1 & 12-Apr-95 & 13 & -152.53 \\
\hline 22-Apr-95 & 13 & 26-Apr-95 & 5 & -54.56 \\
\hline 16-May-95 & 4 & 21-May-95 & 12 & -89.81 \\
\hline 24-Jul-95 & 4 & 26-Jul-95 & 0 & -55.06 \\
\hline 2-Oct-95 & 5 & 11-Oct-95 & 1 & -96.71 \\
\hline $20-$ Oct-95 & 0 & 26-Oct-95 & 6 & -66.28 \\
\hline $30-O c t-95$ & 11 & 4-Nov-95 & 22 & -58.68 \\
\hline 24-Dec-95 & 8 & 25-Dec-95 & 23 & -66.72 \\
\hline 10-Mar-96 & 11 & 14-Mar-96 & 14 & -60.01 \\
\hline 19-Mar-96 & 7 & 24-Mar-96 & 11 & -69.72 \\
\hline 17-Oct-96 & 9 & 26-Oct-96 & 17 & -105.40 \\
\hline 27-Feb-97 & 0 & 4-Mar-97 & 15 & -92.18 \\
\hline 16-Apr-97 & 18 & 21-Apr-97 & 6 & -77.31 \\
\hline 1-May-97 & 17 & 5-Мay-97 & 1 & -66.29 \\
\hline 10-Mar-98 & 11 & 14-Mar-98 & 12 & -116.65 \\
\hline 20-Mar-98 & 10 & 23-Mar-98 & 7 & -87.77 \\
\hline 24-Apr-98 & 1 & 28-Apr-98 & 12 & -73.19 \\
\hline 6-Jun-98 & 16 & 9-Jun-98 & 7 & -50.37 \\
\hline 16-Jul-98 & 3 & 18-Jul-98 & 5 & -58.69 \\
\hline 30-Sep-98 & 15 & 2-Oct-98 & 12 & -57.69 \\
\hline 2-Oct-98 & 14 & 5-Oct-98 & 5 & -55.26 \\
\hline 7-Oct-98 & 0 & 11-Oct-98 & 12 & -67.41 \\
\hline
\end{tabular}

Table 2. List of all ejecta-driven events in the study

\begin{tabular}{lrlrr}
\hline $\begin{array}{l}\text { Start } \\
\text { Date }\end{array}$ & Hour & $\begin{array}{l}\text { End } \\
\text { Date }\end{array}$ & Hour & $\begin{array}{c}\text { Minimum } \\
\text { Dst* }\end{array}$ \\
\hline 6-Feb-95 & 11 & 9-Feb-95 & 9 & -79.23 \\
4-Mar-95 & 12 & 7-Mar-95 & 4 & -91.89 \\
22-Aug-95 & 17 & 23-Aug-95 & 17 & -60.67 \\
18-Oct-95 & 13 & 19-Oct-95 & 19 & -122.96 \\
10-Jan-97 & 5 & 10-Jan-97 & 23 & -76.16 \\
10-Apr-97 & 22 & 11-Apr-97 & 15 & -87.15 \\
21-Apr-97 & 13 & 23-Apr-97 & 4 & -106.66 \\
15-May-97 & 6 & 18-May-97 & 11 & -115.93 \\
26-May-97 & 16 & 29-May-97 & 0 & -72.48 \\
5-Nov-97 & 8 & 9-Nov-97 & 8 & -114.98 \\
10-Dec-97 & 11 & 13-Dec-97 & 1 & -60.14 \\
6-Jan-98 & 16 & 8-Jan-98 & 6 & -76.80 \\
29-Jan-98 & 22 & 31-Jan-98 & 17 & -55.16 \\
17-Feb-98 & 14 & 20-Feb-98 & 10 & -106.35 \\
26-Jun-98 & 0 & 26-Jun-98 & 13 & -100.91 \\
6-Aug-98 & 3 & 9-Aug-98 & 1 & -148.97 \\
20-Aug-98 & 8 & 21-Aug-98 & 8 & -66.47 \\
8-Nov-98 & 21 & 11-Nov-98 & 9 & -142.11 \\
\hline
\end{tabular}

than the CME-driven storm and containing similar integrated epsilon values. Table 3 contains the energy, minimum $D s t^{*}$, and event duration values for these events.

Superposed epoch analyses were conducted for $D s t^{*}, B_{Z}$, solar wind number density, solar wind speed, magnetosonic Mach number, epsilon, ring current injection rate, Joule heating power, and auroral precipitation power. Data were separated into main phase and recovery phase as defined by the minimum $D s t^{*}$ point in each event. Integrated values of the parameters were calculated for each event.

\section{OBSERVATIONS}

Figure 2 shows IMF $B_{Z}$ for CIR and ejecta-driven storms. The CIR storms (Panel A) show rapidly varying $B_{Z}$ which hovers near zero throughout the events, and continues to oscillate rapidly around $B_{Z}=0$ well into the storm recovery. The ejecta storms (Panel B) show a slowly changing $B_{Z}$ that gradually moves from southward to northward orientation and then largely stays northward. Panel $\mathrm{C}$ shows a superposition of $B_{Z}$ for CIR and ejecta-related events. It is clear from the superposition that for the events in this study, when $B_{Z}$ in ejecta-related drivers goes northward it stays there, allowing recovery, while CIR-driven events show a $B_{Z}$ hovering near zero.

Figure 3 shows the superposed solar wind inputs of the entire set of events. Panel A shows the solar wind speed: note the much faster flows during CIR events. The second panel has the epsilon parameter, used as an indicator of input energy. For the storms in this set, the ejecta-related events 
April 16-21, 1997 CIR Event

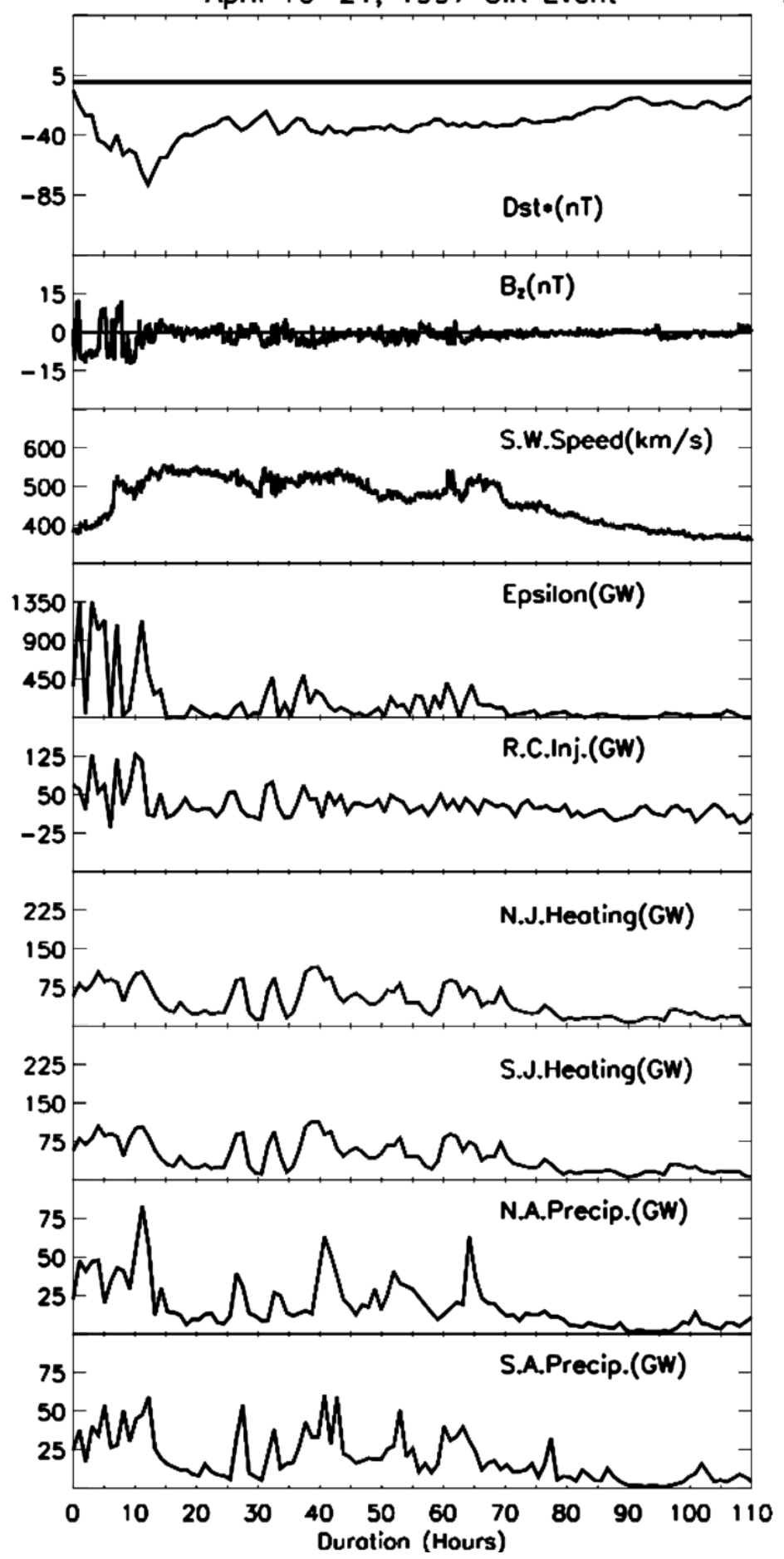

April 21-23, 1997 CME Event

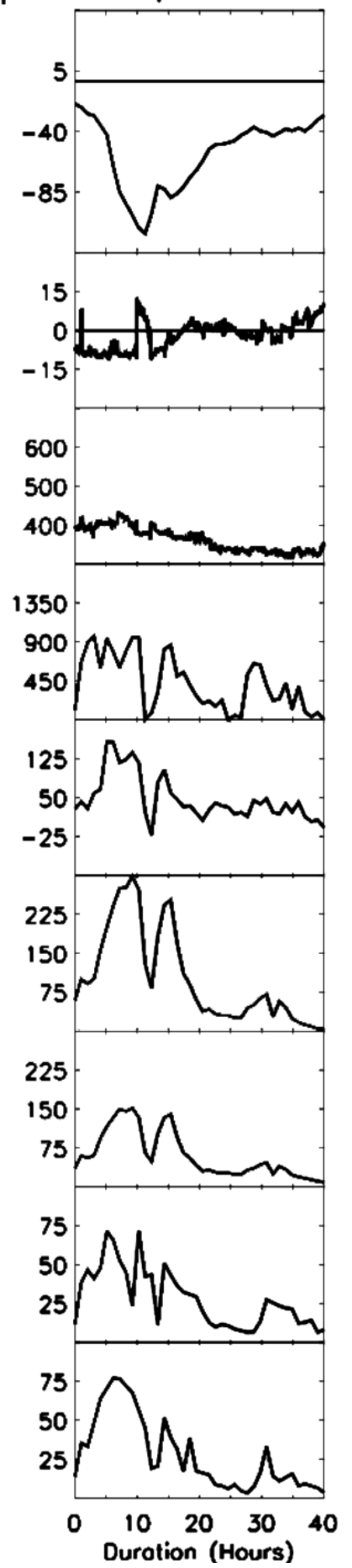

Figure 1. Two example events: a CIR-driven event on April 16-21, 1997 and a CME-driven event on April 21-23, 1997. 
Table 3. Table of energies, minimum $D s t^{*}$, and event durations for example events in Figure 1

\begin{tabular}{|c|c|c|c|c|c|c|c|}
\hline Type of Event & Date & $\begin{array}{l}\text { Duration } \\
\text { (Hours) }\end{array}$ & $\begin{array}{c}\text { Min Dst* } \\
\text { (nT) }\end{array}$ & $\begin{array}{l}\text { Input } \\
\left(10^{15} \mathrm{~J}\right)\end{array}$ & $\begin{array}{l}\text { Ring } \\
\text { Current } \\
\left(10^{15} \mathrm{~J}\right)\end{array}$ & $\begin{array}{c}\text { Joule } \\
\text { Heating } \\
\left(10^{15} \mathrm{~J}\right)\end{array}$ & $\begin{array}{c}\text { Auroral } \\
\text { Precipitation } \\
\left(10^{15} \mathrm{~J}\right)\end{array}$ \\
\hline CIR Event & Apr 16-21, 1997 & 109 & -77.31 & 62.60 & 11.24 & 35.19 & 14.73 \\
\hline Ejecta Event & Apr 21-23, 1997 & 40 & -106.66 & 60.02 & 6.75 & 23.42 & 8.04 \\
\hline
\end{tabular}

show a higher epsilon during the main and early recovery phases. Epsilon peaks about 3 hours before the $D s t^{*}$ minimum, and is nearly double for ejecta what is it for CIRs. The magnetosonic Mach number is plotted in Panel C, and again indicates the much faster flows common to CIR events. Panels D and E show the Joule heating and ring current injection rates, respectively, with both showing only a moderate advantage for ejecta-driven events. Panel F shows the superposed $D s t^{*}$, which shows the clear advantage of ejecta-driven events in producing large $D s t^{*}$ excursions.

Figure 4 shows the total energy output for all studied events. While the input energies are similar for the CIR and ejecta events, the output quantities are larger and outside the error bars for every measured quantity. The duration of the CIR storms, it should also be noted, is substantially larger than for ejecta storms, which could play a role in allowing greater energy deposition and may be a result of continuing driving of the system well into the recovery.

In Figure 5 the averaged energy per hour for all storms in the dataset is shown. The input power for ejecta-driven storms is about double that for CIR-driven storms, and the $D s t^{*}$ is somewhat higher for them as well. The output power for ring current, auroral precipitation, and Joule heating are larger for the ejecta events, but not nearly with the same
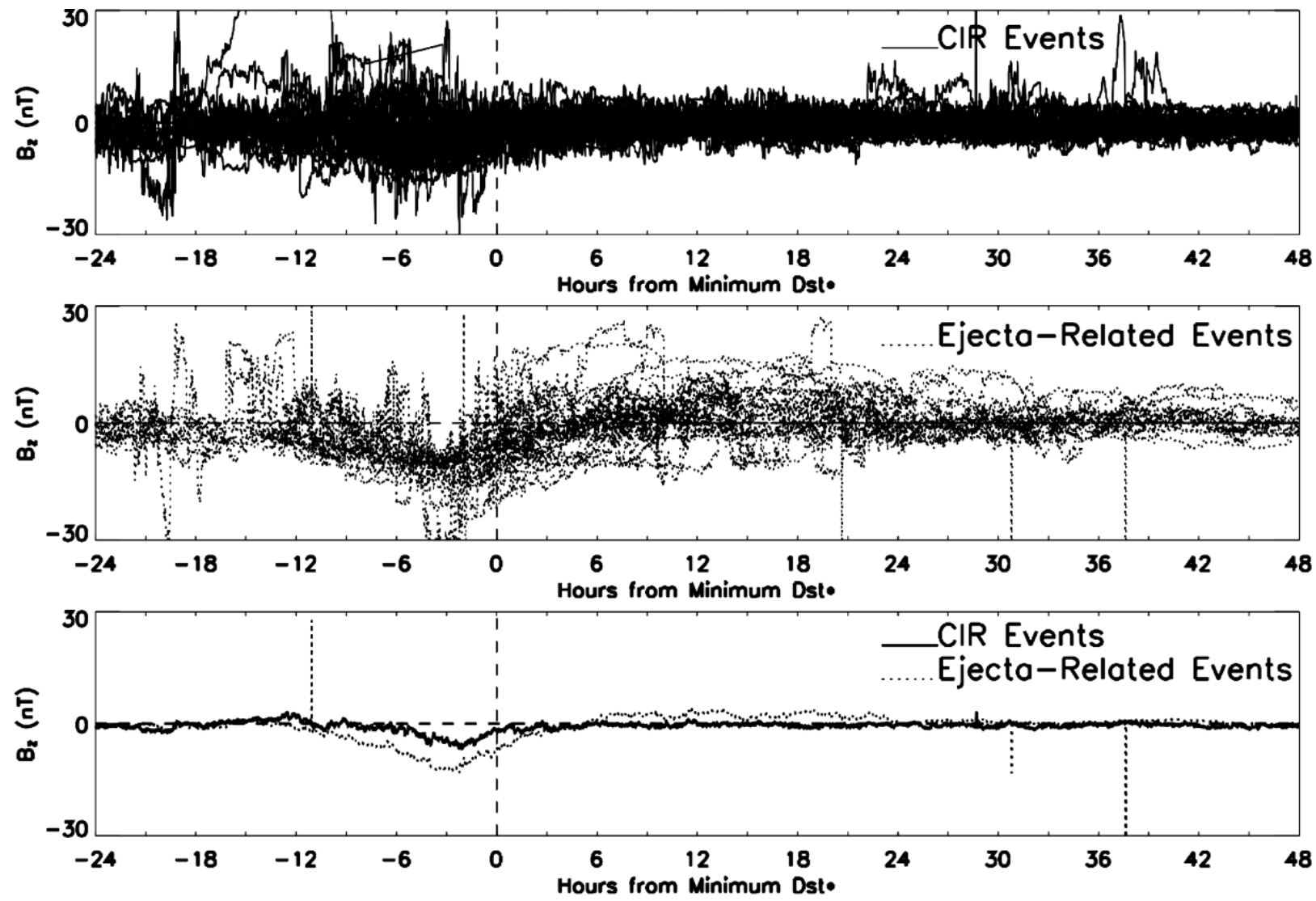

Figure 2. IMF $B_{Z}$ for all events in study. Panel A shows all CIR events, Panel B shows ejecta-related events, and Panel C shows a superposed epoch analysis for all events in the study. Vertical line shows time of minimum $D s t^{*}$. 


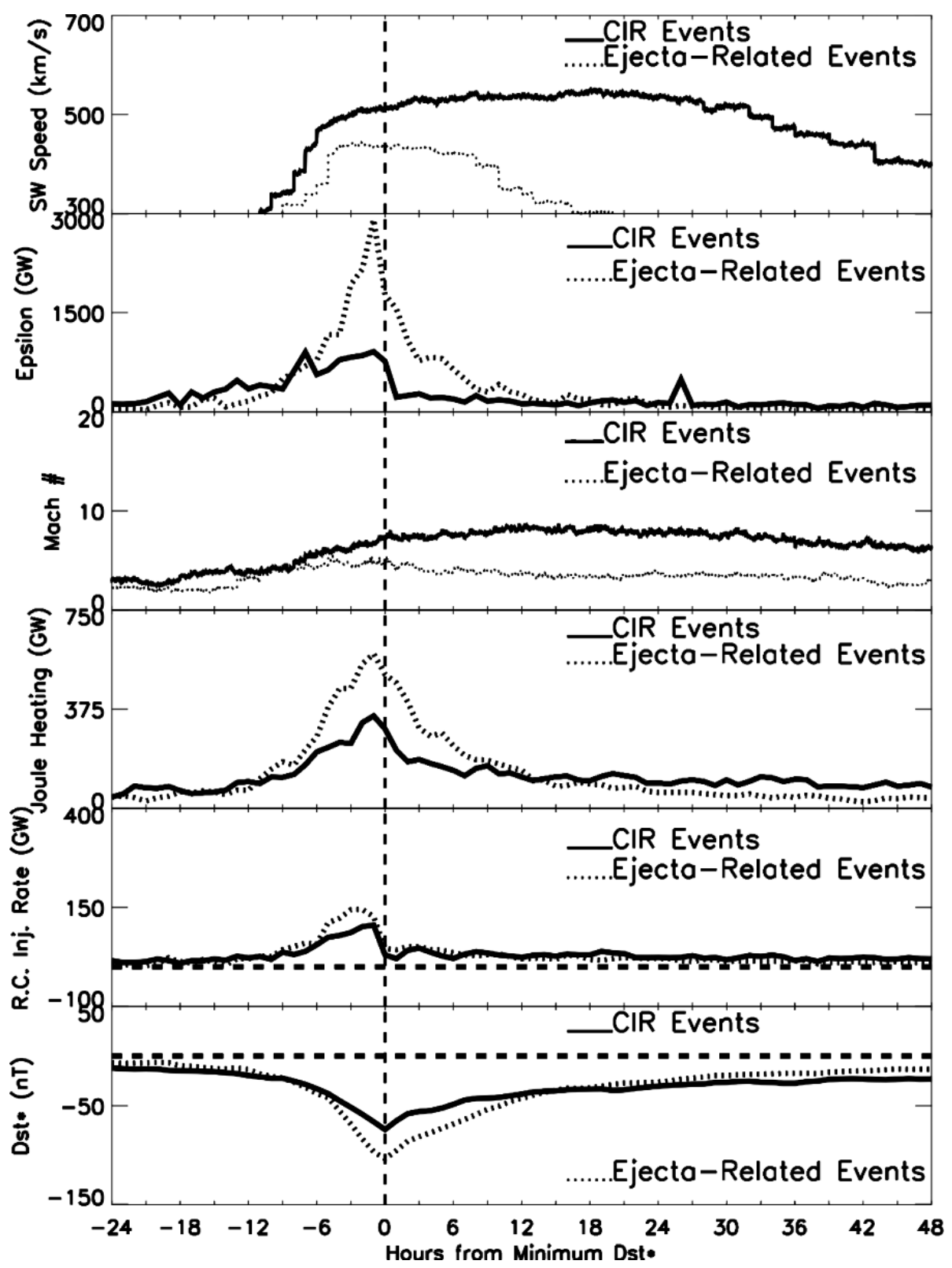

Figure 3. Superposed epoch analysis for solar wind input parameters (Solar wind speed, epsilon, and magnetosonic Mach number) and magnetospheric response (Joule heating, ring current injection rate, and $D s t^{*}$ ) for all events. Vertical line denotes time of minimum $D s t^{*}$.

margin as the input power. This difference in coupling efficiency will become more evident in later figures.

Figure 6 shows the input and output power for the main phase. While the input power for ejecta-driven storms is $70 \%$ larger than for CIR-driven storms, but the ring current injection rate is only $25 \%$ larger, the auroral precipitation rate is almost identical, and the Joule heating rate is $50 \%$ larger.
Figure 7 shows the input and output power for the recovery phase. The input power for recovery phase of the storms in the study averages 2.5 times larger for ejecta-driven than CIR-driven storms, and the ring current injection rate is nearly identical, the auroral precipitation rate is nearly identical, and the Joule heating is 33\% larger. This gives further evidence of stronger coupling efficiency for CIR-driven storms. 


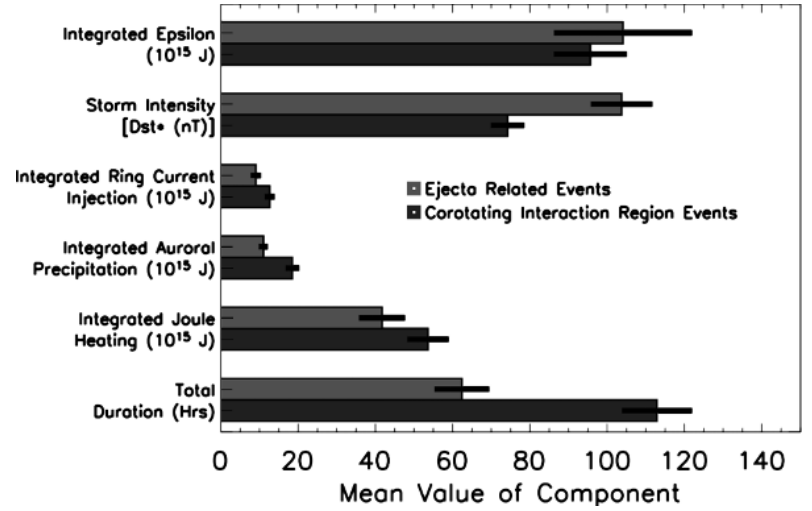

Figure 4. Bar graph depicting storm-integrated solar wind and magnetospheric response parameters for all events.

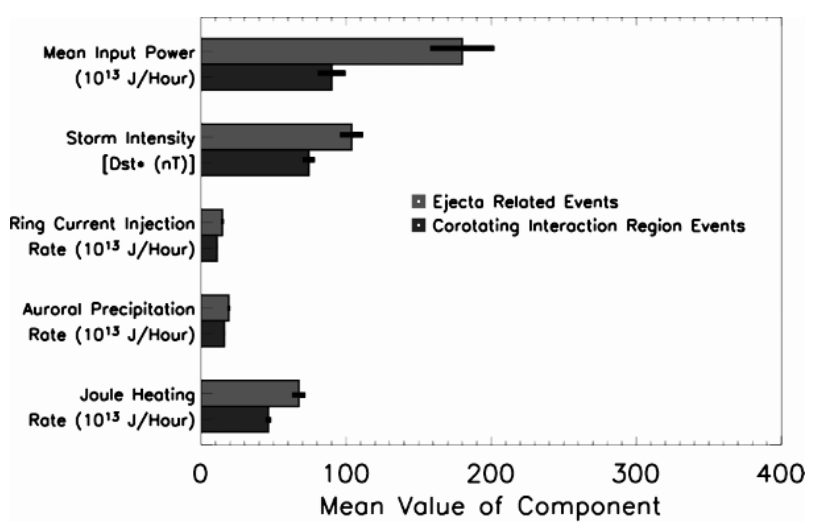

Figure 5. Bar graph depicting values of storm parameters averaged per hour over the storms' duration for all events.

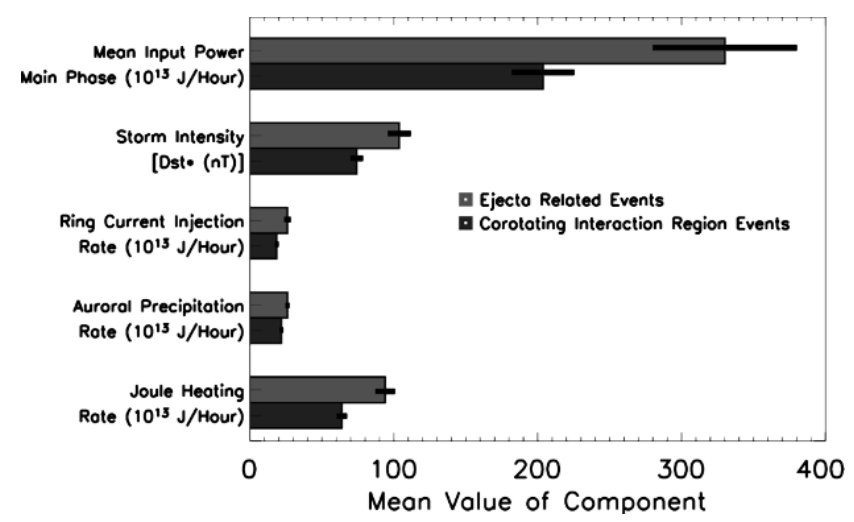

Figure 6. Bar graph depicting values of storm parameters averaged per hour over the storms' main phase for all events.

Figure 8 illustrates in greater detail the differences observed in the preceding plots. In this figure is plotted the output energy on the $y$-axis and input energy on the $\mathrm{x}$-axis. All storms in the dataset are plotted here. Panel A shows the data

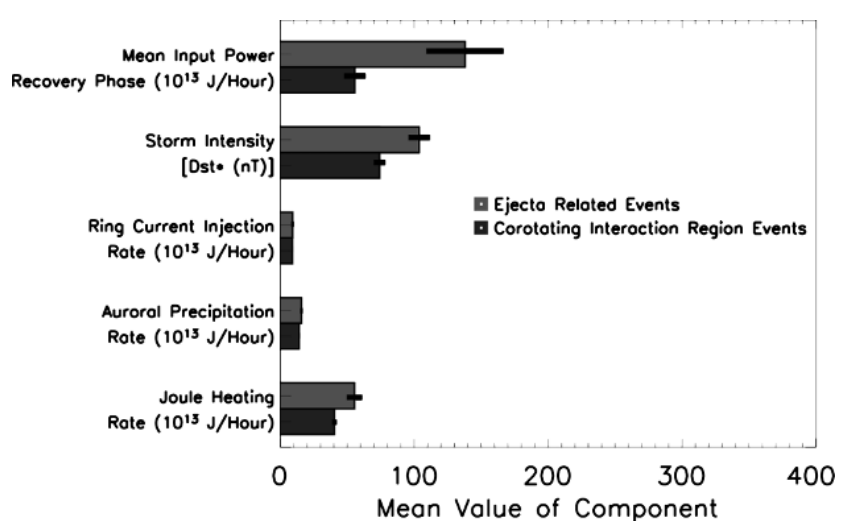

Figure 7. Bar graph depicting values of storm parameters averaged per hour over the storms' recovery phase for all events.
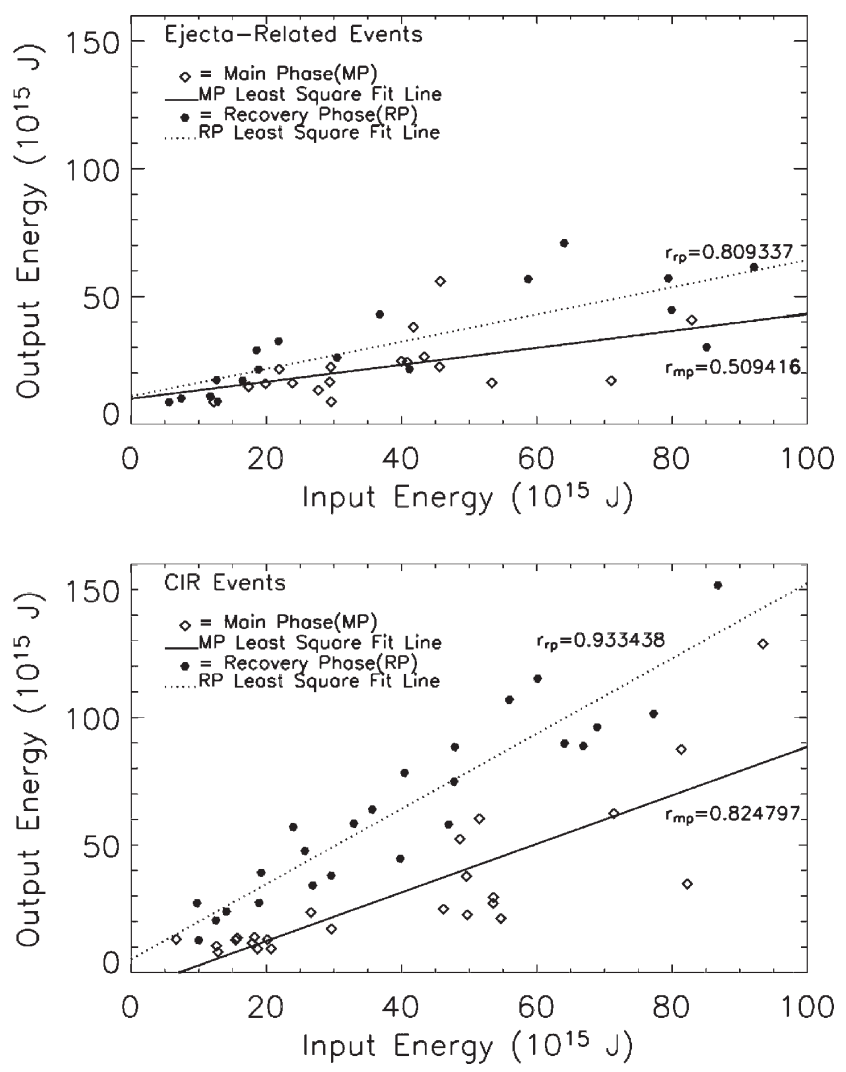

Figure 8. Panel A: Output energy versus input energy (integrated epsilon) for recovery phase and main phase for ejecta-driven storms, shown with linear fit and correlation coefficient for each. Panel B: Same parameters plotted for CIR-driven events.

for ejecta-driven events. Points corresponding to the main phase are indicated as diamonds, while points corresponding to recovery phase are indicated as filled circles. Two least squares fits are shown. The slopes of these lines approximate 
Table 4. Table of energy input, output and geoeffectiveness

\begin{tabular}{|c|c|c|c|c|c|}
\hline Main Phase & $\begin{array}{l}\text { Input } \\
\left(10^{15} \mathrm{~J}\right)\end{array}$ & $\begin{array}{l}\text { Ring Current } \\
\left(10^{15} \mathrm{~J}\right)\end{array}$ & $\begin{array}{c}\text { Joule Heating } \\
\qquad\left(10^{15} \mathrm{~J}\right)\end{array}$ & $\begin{array}{c}\text { Auroral Precipitation } \\
\qquad\left(10^{15} \mathrm{~J}\right)\end{array}$ & Output/Input \\
\hline CIR-Driven & 40 & 4.4 & 17 & 5.7 & 0.68 \\
\hline Ejecta-Driven & 38 & 3.3 & 13 & 3.5 & 0.52 \\
\hline Recovery Phase & $\begin{array}{l}\text { Input } \\
\left(10^{15} \mathrm{~J}\right)\end{array}$ & $\begin{array}{l}\text { Ring Current } \\
\qquad\left(10^{15} \mathrm{~J}\right)\end{array}$ & $\begin{array}{c}\text { Joule Heating } \\
\qquad\left(10^{15} \mathrm{~J}\right)\end{array}$ & $\begin{array}{c}\text { Auroral Precipitation } \\
\qquad\left(10^{15} \mathrm{~J}\right)\end{array}$ & Output/Input \\
\hline CIR-Driven & 40 & 8.2 & 36 & 12 & 1.39 \\
\hline Ejecta-Driven & 39 & 3.3 & 18 & 4.8 & 0.67 \\
\hline
\end{tabular}

the coupling efficiency for main and recovery phases of storms. The slopes of these lines are very different, showing a much greater coupling efficiency for the CIR storms than for the ejecta storms, particularly in the recovery phase.

Table 4 shows the energy input and output for all events. The last column shows the output/input, which is a way to quantify the coupling efficiency. From this measure, the CIRdriven storms show a higher geoeffectiveness than do the ejecta-driven storms.

Figure 9 illustrates the recovery phase energies for storms in different stages of recovery. Panel A shows 20\% recovery of the initial $D s t^{*}$ excursion value, Panel B shows $40 \%$, and Panel C shows $80 \%$. The trend of CIR-driven storms containing greater output energies in the recovery phase is consistent throughout the panels.

\section{DISCUSSION}

\subsection{Solar Wind Driving Conditions}

From the $B_{Z}$ plots in Figure 2 it is clear that the solar wind driving conditions are substantively different in CIRs than ejecta-related events. In the events studied the IMF $B_{Z}$ moves, on average, towards a more northward configuration over time in CIRs, but keeps oscillating about $B_{Z}=0$ for a long duration, typically days. These long-lasting variations could have the effect of driving the magnetosphere long after the main CIR-driven storm has begun to recover. Since it moves repeatedly back into southward $B_{Z}$ configurations, sporadic reconnection may be driven and energy coupling may be correspondingly enhanced. The ejecta-related events typically show a clearer cutoff to the southward $B_{Z}$ and the resulting storms appear to recover with less interruption.

During the CIR-driven storms' recovery phases, highintensity long-duration continuous AE activity (HILDCAA) events are known to occur [e.g., Tsurutani et al., 2004]. These events are a result of the continual driving of the storms during the recovery phase by high-speed solar wind streams and they are evident in the very slow recovery of $D s t$ for these events. It is believed that one mechanism of energy transfer during these events involves reconnection associated with the southward components of large amplitude interplanetary Alfvén waves that are present in high-speed streams [Tsurutani et al., 2006 (this volume)].

Another aspect that may affect the coupling efficiency of CIRs is the high solar wind speed itself. As Figure 3 shows, the CIRs have, on average, markedly higher solar wind speeds. As has been pointed out by Lu et al. [1998], the kinetic energy flux is much greater than the Poynting flux for typical solar wind conditions. For example, $\mathrm{Lu}$ et al. [1998] found the kinetic power to be two orders of magnitude higher than the electromagnetic input (epsilon parameter) for the January 1997 storm. So if even a small percentage of kinetic energy flux is important in driving the magnetosphere, it could produce a large effect. Secondly, this also produces an increased magnetosonic Mach number, which may then increase the reconnection rate, thereby coupling more energy into the magnetosphere-ionosphere system. These important differences in the driving conditions may be responsible for enhanced efficiency of energy coupling from CIRs relative to ejecta.

\subsection{Geoeffectiveness}

Geoeffectiveness was extensively analyzed for the events in this study. It is clear from the bar graphs in Figure 4 that CIRs are more efficient at coupling energy into the magnetosphere than ejecta events. Even with the same input energy available to the system (epsilon), the output was measurably higher in every energy sink evaluated.

Since the CIR-driven events last typically so much longer than the ejecta events, the possibility that this longer duration was the cause of increased output was considered. Since ejecta storms typically produce large $D s t^{*}$ excursions (see Figures), it could be the case that they couple more energy faster and the CIRs "catch up" only over the long duration of those storms. As seen in Figures 5, 6, 7, and 9, this was not found to be the case. If anything, the power output for CIR and ejecta events looked very similar, so the total energy 

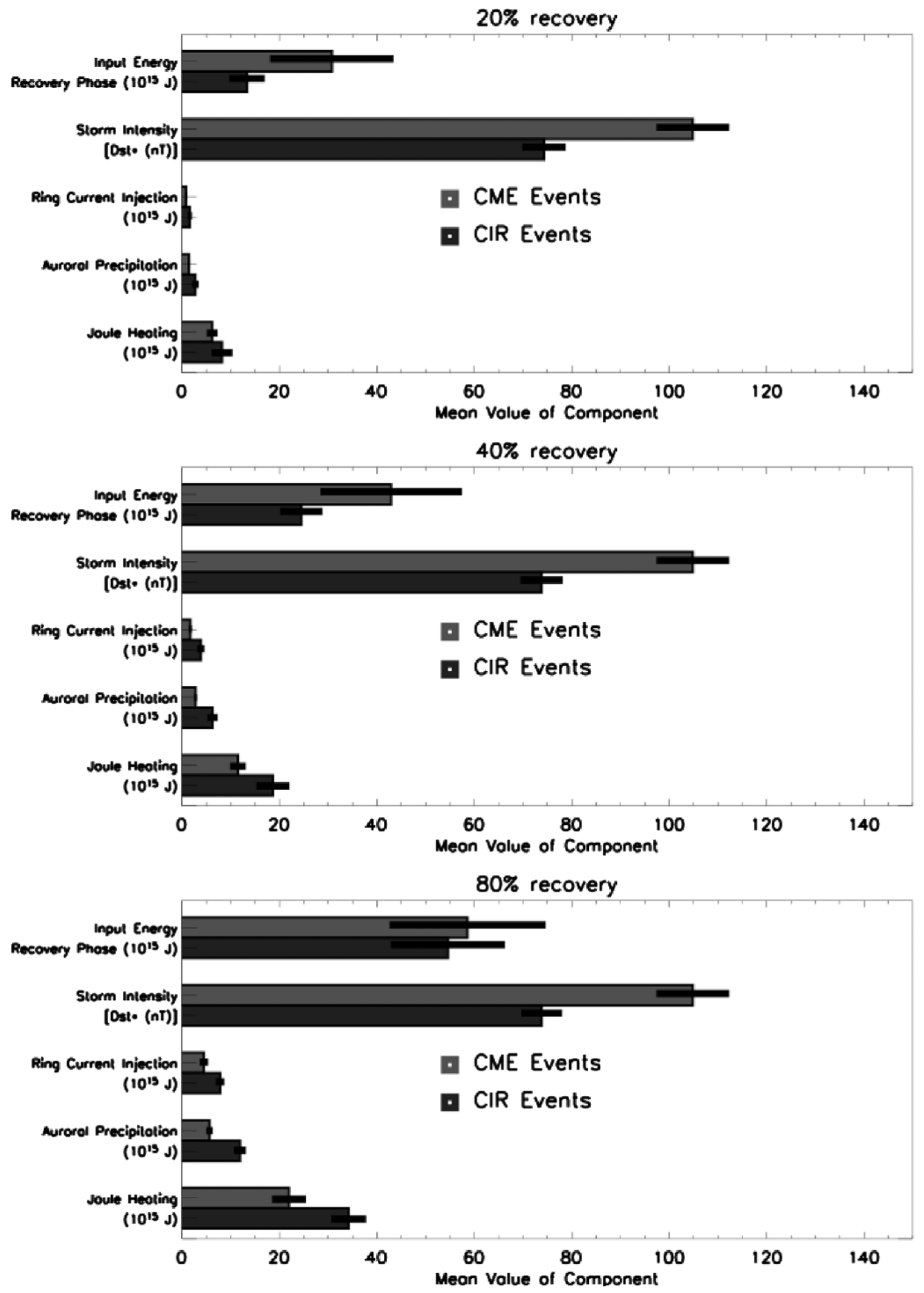

Figure 9. Bar graphs depicting storm parameters for different stages of recovery in all events in the study. Panel A: Values for storms after $20 \%$ of $D s t^{*}$ recovery. Panel B: Values for storms after $40 \%$ of $D s t^{*}$ recovery. Panel C: Values for $80 \%$ of $D s t^{*}$ recovery. 
output is higher for CIRs and the per-hour values are similar for both in the main phase. In Figure 9, further confirmation of this is seen as the trends remain for all stages of recovery.

Table 4 shows the output energy divided by the input energy, as an estimate of the coupling efficiency. Clearly, the CIRs are more geoeffective in these events than are the CMEs, and particularly so in the recovery phase.

The fact that the output/input for recovery phase in CIRdriven storms is greater than 1.0 indicates again the limitations of the epsilon parameter, as clearly the output cannot exceed the input. This indicates either that the Poynting flux was underestimated by epsilon or that there is a measurable influence from another type of energy coupling, for example the kinetic energy flux.

\subsection{Recovery Phase Energy Coupling}

It is in the recovery phase where the dynamics of CIR and ejecta storms diverge the most. Figure 7 shows the power output from both types of storms in recovery phase, where full recovery is considered to be when the $D s t^{*}$ recovers to $80 \%$ of its initial value. Even though the estimated input power for ejecta-driven storms is about 2.5 times that for CIRs, the output power is very similar. This greater coupling efficiency appears as a consistent pattern, as shown clearly in Figures 8 and 9 and Table 4.

From Figure 8 it is clear that the recovery phase behavior of CIR and ejecta-driven storms is very different. Both the clear correlation between input and output energy in the recovery phase and the high geoeffectiveness suggest that the phase of the storm that exists after the $D s t^{*}$ minimum in a CIR-driven event may not be a pure recovery. This may be due to the repeated excursions into southward $B_{Z}$ that are characteristic of the solar wind conditions in CIR events. Ejecta-driven events showed weaker responses to recoveryphase driving, which likely accounts for their faster recovery and overall shorter duration.

\section{CONCLUSIONS}

From this study, it has been shown that the solar windmagnetosphere energy coupling of CIR-driven and ejectadriven storms differ in several important properties. In particular, while the ejecta-driven events typically produce greater Dst excursions than the CIR events, the CIRs are more geoeffective in the sense of greater overall energy output than are the ejecta events. When CIR and ejecta events with the same input energies are compared, CIR events have greater energy output. Output power is similar for the two types of events in the storms' main phases, but in recovery phase the CIRs have less input power and the similar output power to ejecta events. More specifically, the measured geoeffectiveness during the recovery phases of CIR-driven storms is double that of ejecta-driven events. These key differences, especially in the recovery phase, are likely due to the rapidly oscillating IMF $B_{Z}$ that is typical of CIR-driven events well into storm recovery and the energy coupling that produces.

Acknowledgments. The authors acknowledge Ian Richardson for identification of CIR and ejecta events. Work of NT and EM is supported by NSF Career Grant ATM0454685. EM was also supported by the NIH MARC program. The CEDAR database is sponsored by the NSF. Intersatellite calibration work by BE is supported by National Space Weather Program Grant \# 0208145. NT acknowledges fruitful discussions and inspiration from the Chapman Conference on Corotating Solar Wind Streams and Recurrent Geomagnetic Activity. The authors also acknowledge OMNIWeb and the Kyoto Geophysical Data Center for making solar wind and Dst data available and convenient, and gratefully thank the WIND instrument teams for making their data available for use. Finally, the authors thank the reviewers for their valuable comments.

\section{REFERENCES}

Baker, D.N., N.E. Turner, and T.I. Pulkkinen, Energy transport and dissipation in the magnetosphere during geomagnetic storms, J. Atmos. SolarTerr. Phys., 63, 421-429, 2001

Bargatze, L.F., R.L. McPherron, and D.N. Baker, Solar wind-magnetosphere energy input functions, in Solar Wind-Magnetosphere Coupling, Edited by Y. Kamide and J.A. Slavin, 101-109, 1985.

Campbell, W.H., Geomagnetic storms, the Dst ring current myth and lognormal distributions, J. Atmos. Solar-Terr. Phys., 58, 1171-1187, 1996.

Chun, F.K., D.J. Knipp, M.G. McHarg, G. Lu, B.A. Emery, S. Vennerstrom, O.A. Troshichev, Polar cap index as a proxy for hemispheric Joule heating, Geophys. Res. Lett., 26, 1101-1104, 1999.

Dessler, A.J., and E.N. Parker, Hydromagnetic theory of magnetic storms, J. Geophys. Res., 64, 2239-2259, 1959.

Echer, E. and W.D. Gonzalez, Geoeffectiveness of interplanetary shocks, magnetic clouds, sector boundary crossings and their combined occurrence, Geophys. Res. Lett., 31, doi:10.1029/2003GL019199, 2004.

Emery, B.A., D.S. Evans, M.S. Greer, K. Kadinsky-Cade, E. Holeman, F.J. Rich and W. Xu, NOAA and DMSP Intersatellite Adjusted Hemispheric Power Data Sets, http://cedarweb.hao.ucar.edu and http:// cedarweb.hao.ucar.edu/instruments/ehp.html, Coupling, Energetics and Dynamics of Atmospheric Regions (CEDAR) Database at the National Center for Atmospheric Research (NCAR), Boulder, Colorado, USA, 2005.

Emery, B.A., D.S. Evans, M.S. Greer, K. Kadinsky-Cade, E. Holeman, F.J. Rich and $\mathrm{W}$. $\mathrm{Xu}$, The low energy auroral electron and ion hemispheric power after NOAA and DMSP intersatellite adjustments, NCAR Scientific and Technical Report, TN-470 + STR, 2006.

Feldstein, Y.I., A.E. Levin, J.U. Kozyra, B.T. Tsurutani, A. Prigancova, L. Alperovich, W.D. Gonzalez, U. Mall, I.I. Alexeev, L.I. Gromova, and L.A. Dremukhina, Self-consistent modeling of large-scale distortions in the geomagnetic field during the 24-27 September 1998 major magnetic storm, J. Geophys. Res., 110, A11214, doi:10.1029/2004JA010584, 2005.

Gonzalez, W.D., B.T. Tsurutani, A.L.C. Gonzalez, E.J. Smith, F. Tang, and S.-I. Akasofu, Solar wind-magnetosphere coupling during intense magnetic storms (1978-1979), J. Geophys. Res., 94, 8835-8851, 1989.

Gonzalez, W.D., B.T. Tsurutani, and A.L. Clúa de Gonzalez, Interplanetary origin of geomagnetic storms, Space Science Reviews, 88: 529-562, 1999. 
Huttunen, K.E., H.E. Koskinen, R. Schwenn, Variability of magnetospheric storms driven by different solar wind perturbations, J. Geophys. Res., 107, doi:10.1029/2001JA900171, 2002.

Knipp, D.J., B.A. Emery, M. Engebretson, X. Li, A.H. McAllister, T. Mukai, S. Kokubun, G.D. Reeves, D. Evans, T. Obara, X. Pi, T. Rosenberg, A. Weatherwax, M.G. McHarg, F. Chun, K. Mosely, M. Codrescu, L. Lanzerotti, F.J. Rich, J. Shriver, and P. Wilkinson, An overview of the early November 1993 geomagnetic storm, J. Geophys. Res., 103, 26197, 1998.

Knipp, D.J., W.K. Tobiska, and B.A. Emery, Direct and indirect thermospheric heating sources for solar cycles 21-23, Solar Physics, 224, 495-505, 2004.

Lu, G., D.N. Baker, R.L. McPherron, C.J. Farrugia, D. Lummerzheim, J.M. Ruohoniemi, F.J. Rich, D.S. Evans, R.P. Lepping, M. Brittnacher, X. Li, R. Greenwald, G. Sofko, J. Villain, M. Lester, J. Thayer, T. Moretto, D. Milling, O. Troshichev, A. Zaitzev, V. Odintzov, G. Makarov, and K. Hayashi, Global energy deposition during the January 1997 magnetic cloud event, J. Geophys. Res., 103, 11,685-11,694, 1998.

Monreal-MacMahon, R. and W.D. Gonzalez, Energetics during the main phase of geomagnetic superstorms, J. Geophys. Res., 102, 14,199-14,207, 1997.

O'Brien, T.P., and R.L. McPherron, An empirical phase space analysis of ring current dynamics: Solar wind control of injection and decay, $J$. Geophys. Res., 105, 7707-7719, 2000.

O’Brien, T.P., R.L. McPherron, and M.W. Liemohn, Continued convection and the initial recovery of Dst, Geophys. Res. Lett., 29(23) 2143, doi:10.1029/2002GL015556, 2002.

Ohtani, S., M. Nosé, G. Rostoker, A.T.Y. Lui, and M. Nakamura, Stormsubstorm relationship: Contribution of the tail current to Dst, J. Geophys. Res., 106, 21999-21209, 2001.

Perreault, P., and S.-I. Akasofu, A study of geomagnetic storms, Geophys. J. R. Astr. Soc., 54, 547, 1978.

Pulkkinen, T.I., N. Ganushkina, D.N. Baker, N.E. Turner, J.F. Fennell, J. Roeder, T.A. Fritz, M. Grande, B. Kellett, G. Kettmann, Ring current ion composition during solar minimum and rising solar activity: Polar CAMMICE/MICS results, J. Geophys. Res., 106, 19131-19147, 2001.
Sckopke, N., A general relation between the energy of trapped particles and the disturbance field near the Earth, J. Geophys Res., 71, 3125-3130, 1966.

Tsurutani, B.T., W.D. Gonzalez, F. Guarnieri, Y. Kamide, X. Zhou, and J.K. Arballo, Are high-intensity long-duration continuous AE activity (HILDCAA) events substorm expansion events?, J. Atmos. \& Solar-Terr. Phys., 66, 167-176, 2004.

Tsurutani, B.T., N. Gopalswamy, R.L. McPherron, W.D. Gonzalez, G. Lu, F.L. Guarnieri, Magnetic storms caused by corotating solar wind streams, AGU Geophysical Monograph Series, this volume, 2006.

Turner, N.E., D.N. Baker, T.I. Pulkkinen, and R.L. McPherron, Evaluation of the tail current contribution to Dst, J. Geophys. Res., 105, 5431-5439, 2000a.

Turner, N.E., Solar wind-magnetosphere coupling and global energy budgets in the Earth's magnetosphere, Doctoral Dissertation, $2000 \mathrm{~b}$.

Turner, N.E., D.N. Baker, T.I. Pulkkinen, J.L. Roeder, J.F. Fennell, and V.K. Jordanova, Energy content in the storm time ring current, J. Geophys. Res., 106, 19149-19156, 2001.

Vichare, G., S. Alex, and G.S. Lakhina, Some characteristics of intense geomagnetic storms and their energy budget, J. Geophys. Res., 110, A03204, doi:10.1029/2004JA010418, 2005.

Weiss, L.A., P.H. Reiff, J.J. Moses, R.A. Heelis, and B.D. Moore, Energy dissipation in substorms, in Substorms 1, edited by B. Hultqvist and S. Akasofu, 309-318, 1992.

Zhang, J., M.W. Liemohn, J.U. Kozyra, B.J. Lynch, and T.H. Zurbuchen, A statistical study of the geoeffectiveness of magnetic clouds during high solar activity years, J. Geophys. Res., 109, A09101, doi:10.1029/ 2004JA010410, 2004.

Niescja E. Turner and Elizabeth J. Mitchell, Physics and Space Sciences, Florida Institute of Technology, Melbourne, Florida 32901

Delores J. Knipp, Physics Department, US Air Force Academy, USAFA, Colorado 80840

Barbara A. Emery, High Altitude Observatory, National Center for Atmospheric Research, Boulder, Colorado 80307 